\title{
Modelling children's independent territorial range by discretionary and nondiscretionary trips
}

\author{
Samia Sharmin \\ Queensland University of Technology \\ samia.sharmin@connect.qut.edu.au \\ Md. Mazharul Haque \\ Queensland University of Technology \\ m1.haque@qut.edu.au
}

\author{
Md. Kamruzzaman \\ Monash University \\ md.kamruzzaman@monash.edu
}

\begin{abstract}
The decline of children's independent mobility (CIM) is now a global concern. This study aims to identify the determinants of the territorial range (TR) of CIM, i.e., the geographical distance between home and places where children are allowed to wander. TR for both discretionary and nondiscretionary trips is studied based on data collected through a questionnaire survey, travel diary, and mapping of travel routes. The study sample was comprised of 151 children 9-14 years of age from Dhaka, Bangladesh. Built environment (BE) data were collected/derived through walkability audits of children's walking routes and spatial analyses. Children's TR was regressed by BE, sociodemographics, and perceptual factors. Three multiple regression models were estimated: overall TR, discretionary TR, and nondiscretionary TR. Results showed that children had a longer TR for nondiscretionary trips $(664.14 \mathrm{~m})$ compared to discretionary trips $(397.9 \mathrm{~m})$. Discretionary TR was largely explained by angular step-depth, street connectivity and the condition of the walking environment of the taken routes. In contrast, angular step-depth, the presence of commercial and retail land uses and the condition of the walking environment were found to be significant predictors of nondiscretionary TR. Children's perception of social and physical dangers and their satisfaction with tree coverage in the neighborhood also influenced their TR. The findings can inform measures to be taken to expand TR in the urban environment.
\end{abstract}

Keywords: Territorial range, children independent mobility, space syntax, built environment, perception of local environment

\section{Introduction}

A large body of the literature affirms the scholarly interest in children's independent mobility (CIM) the freedom of children to travel around their own neighborhood without adult accompaniment

Copyright 2021 Samia Sharmin, Md. Kamruzzaman \& Md. Mazharul Haque

http://dx.doi.org/10.5198/jtlu.2021.1889

ISSN: 1938-7849 | Licensed under the Creative Commons Attribution - Noncommercial License 4.0

The Journal of Transport and Land Use is the official journal of the World Society for Transport and Land Use (WSTLUR) and is published and sponsored by the University of Minnesota Center for Transportation Studies.

\section{Article history:}

Received: September 1, 2020

Accepted: November 28, 2020

Available online: March 15,

2021 
(Tranter \& Whitelegg, 1994). In addition to overall health benefits (physical, mental, emotional etc.), CIM has some broader influences on city and society, such as environmental sustainability, community liveability, informal social control, and parents' reduced chauffeuring time and mental stress (Gershuny, 1993; Prezza, Alparone, Renzi, \& Pietrobono, 2009; Tranter \& Pawson, 2001; U.S. Environmental Protection, 2003).

CIM has been conceptualized and measured in various ways, such as territorial range (TR) - the maximum distance that children travelled independently, mobility licence - parental permission to go somewhere independently, independent time spent outside, and number of destinations visited independently. Among these, TR is the earliest, easily understandable and established measure (Hillman, Adams, \& Whitelegg, 1990; Van Vliet, 1983). TR can also be described as a "transforming mechanism through which children interact with and learn about their local environment" (Spilsbury, 2005, p. 81). It is a key to a child's physical, social and cognitive development, including the acquisition of mapping/ spatial skills (Connolly \& Bruner, 1974).

Considering the importance attached to TR, a shrinkage of it has been identified as a major visible transformation among children worldwide over the last few decades. In Australia, for example, 32\% of children aged 8-12 years walk or cycle independently less than $100 \mathrm{~m}$ from home (Veitch, Salmon, \& Ball, 2008). On the other hand, in England, 35\% of 7-11 years old children are allowed to travel $500 \mathrm{~m}$ independently from home (Shaw et al., 2013). More than 20 years ago, a UK study highlighted the dramatic loss in child's independent TR from $10 \mathrm{~km}$ in 1919 to $300 \mathrm{~m}$ in 1990 (Hillman et al., 1990). Concomitantly, a shrinkage of activity domain of children has also been evident. A recent study in Canada reported that $15 \%$ of $9-13$ years old children did not venture more than $200 \mathrm{~m}$ from their home independently (Loebach \& Gilliland, 2014). A shrinkage of TR is greater for children living in urban environments than that of in rural/suburban areas (Carver, Panter, Jones, \& van Sluijs, 2014; Van Vliet, 1983).

A number of studies have identified different determinants of TR (Carver et al., 2014; Fyhri, Hjorthol, Mackett, Fotel, \& Kyttä, 2011; Prezza, Alparone, Cristallo, \& Luigi, 2005; Van Vliet, 1983). These studies have shown that the built environment $(\mathrm{BE})$ is a major determinant of TR. BE is measured by a range of indicators in these studies ranging from land uses (e.g., residential, commercial, mixed-use, recreational etc.) to street design (e.g., street width, intersection density, road density, walkability, effective walkable area etc.) (Carver et al., 2014; Loebach \& Gilliland, 2014; Monsur \& Islam, 2011). However, the BE-TR studies largely overlooked the syntactic BE despite its growing application in children's active transport research (Argin \& Torun, 2015; Helbich et al., 2016; Ozbil, Argin, \& Yesiltepe, 2016; Zdrahalova \& Boumova, 2016).

Syntactic BE (based on space syntax concept) focuses on the topology of the spatial environment, based on the assumption that pedestrian movement is largely shaped by the configuration of a road network and the visual distance (defined as "depth") between the origin and destination of a route. This is in contrast to the conventional geographic approach, which assumes that pedestrian movement is shaped by the metric distance and land use properties (Hillier, 1998; Hillier \& Hanson, 1984). Previous studies exploring children's interaction with the syntactic properties of BE identified numerous influential measures (e.g., integration - the closeness of each street segment to all other segements, choice - chance of a segment to fall on the shortest path between many origins and destinations). For instance, children's walking to school is negatively associated with "integration" (Argin \& Torun, 2015) and positively associated with "choice" (Helbich et al., 2016). On the other hand, Zdrahalova and Boumova (2016) found no such relation between these measures and children's walking. These contradictory findings indicate the need for further research to explore the potential of syntactic properties of BE in promoting TR. In addition, existing studies focused on area-based BE indicator (e.g., presence of footpath, number of tree 
shades, benches, aesthetic and comfort) rather than deriving these indicators along travel routes where walking really occurs.

Most previous studies measured TR by combining school and non-school ranges for a general understanding of CIM. Despite the differences between discretionary (e.g., park, shops) and non-discretionary (e.g., school) trips in terms of their importance in daily life (Fyhri et al., 2011), comparative studies between discretionary and nondiscretionary TR are scarce. The reason for TR being destination specific (i.e., children would walk longer distances independently in some destinations but not in other destination) is that when certain activities are done regularly (non-discretionary activities such as school), children get the opportunity to know the environment better, which builds confidence to the parents and the kids.

It is also known that children's perception of the barriers/risks presented by the local environment, whether evidence-based or not, restricts overall CIM. However, limited TR studies have explored children's perception of BE in comparison to their parents' perception (Ghekiere et al., 2016; Loebach \& Gilliland, 2014; Schoeppe, Duncan, Badland, Rebar, \& Vandelanotte, 2016). These studies found that traffic and social safety are major concerns for children. In Canada, for example, children of 9-13 years old who perceived lower levels of neighborhood risk travelled significantly longer $(1048.4 \mathrm{~m})$ than those who perceived a higher level of risk $(793.9 \mathrm{~m})$ (Loebach \& Gilliland, 2014). So far, very few, if any, studies have examined the perceptions of walking environment, including the physical appearance of the route on TR. Özbil Torun (2015) argued that children's perception of neighborhood is facilitated by the spatial configuration and urban design qualities and affects their route selection to school. Since children are involved around decision making of their independent travel, their perception to local environment should be taken into account.

In light of the above research gaps, this study seeks to contribute to knowledge by answering two research questions: (1) What is the extent of TR for discretionary and nondiscretionary activities, and do they differ significantly from the overall TR?; (2) How are discretionary and nondiscretionary TRs affected by BE, socio-demographic and perceptual factors?. Section 2 presents the methodology applied to answer the above questions. The results are presented in Section 3, which are discussed in policy terms in Section 4. Section 5 presents the strengths and limitation of this study in light of other studies on this topic.

\section{$2 \quad$ Methodology}

The study answers the research questions using data collected from Dhaka, Bangladesh. Dhaka was selected because there is a scarcity of CIM research in the context of developing countries (Sharmin $\&$ Kamruzzaman, 2017). The study employed multiple instruments including questionnaire survey for travel information and socio-demographic data from children and parents, and walkability audit for route level BE data. Area-based spatial data (e.g., route network) were collected from the Capital Development Authority of Bangladesh. The study design is briefly described below.

\subsection{Recruitment of participants and questionnaire survey}

Children and parents were recruited via schools (151 children aged 10-14 years and 151 parents) across Dhaka, Bangladesh. A two-phase random selection of schools was conducted due to the unavailability of the complete school list. At the first phase, four government schools were randomly selected using the Bangladesh Bureau of Educational Information and Statistics (BANBEIS) and at the second phase, eight private schools from the areas of previously selected schools were included using information from 
Google map. Selected schools were approached with an approval letter from the ministry of education, Bangladesh, of which eight agreed to participate. During the school visit by one of the researchers, children of respective age were informed about the objective and potential outcome of the study. Children received an invitation letter with a consent form for them and their parent to participate in a questionnaire survey. Upon the return of the signed consent from parents, parents were contacted over the phone for an appointment to conduct a questionnaire survey at their homes. Children who were able to produce consent from their parents were only recruited due to ethical consideration - this left out the orphans from the survey. This study included children living within a $1600 \mathrm{~m}$ buffer of selected schools known as a walkable buffer for the selected aged group (McDonald \& Aalborg, 2009). School visit and data collection were conducted during September - December 2017.

The questionnaire survey was conducted in two phases: child survey and parent survey. At first, children reported their age, gender, individual factors (e.g., cycle ownership, number of friends) and perception of their neighborhood environments (e.g., safety, aesthetics). They also completed a two-day travel diary (last weekday and weekend/day-off) where they provided travel information including visited destinations, mode of transport and accompaniment of trips. Finally, they documented the origin and destinations of their trips including taken routes on a provided Google map. The school-based children recruitment and instrument applied for data collection from children (i.e., travel diary and mapping- activities) were adopted from previous TR studies (Fagerholm \& Broberg, 2011; Loebach \& Gilliland, 2014; Villanueva, Giles-Corti, Bulsara, Timperio, et al., 2012). The perceived BE characteristics were assessed by eight questions developed based on Villanueva, Giles-Corti, Bulsara, McCormack, et al. (2012) and Page, Cooper, Griew, and Jago (2010) which showed good reliability and validity. Initially, a 5-point Likert scale ( $1=$ strongly disagree, $3=$ strongly agree) was developed for perception data collection, however, the pilot survey (11\% of total participants) revealed that children faced difficulties to deal with a 5-point scale which eventually ended up adopting 2-point dichotomous scale $(0=$ disagree, $1=$ agree) for the final survey. Children were surveyed with their parent's presence. After the completion of the child's survey, a respective parent reported socio-demographic information of the family (e.g., age and education of parents, household income, ownership of motorised vehicle etc.)

Table 1 presents summary statistics of participants' socio-demographic variables. Overall, 130 ( $86 \%$ of the original sample) children with a mean age of 12.38 years reported at least one independent trip. The proportion of boys and girls were equal. Majority of children reported having no cycle (63.1\%). $73.1 \%$ and $78.5 \%$ of children respectively reported having lots of friends and known people in the neighborhood. Most households (45.4\%) had an income of taka 26000-50000 (\$325-\$625) and no access to motorised vehicles (mean $=0.25$, range $=0-3)$. Moms $(84.6 \%)$ and dads $(56.9 \%)$ mostly aged between 30-45 years. The majority of moms having a higher education of class 12 (36.2\%). On the other hand, about $39.2 \%$ of dads had a bachelor/master's degree.

A majority of children indicated that they like to roam around their neighborhood actively (85.4\%) because they perceived neighborhood is safe in terms of traffic (63.1\%), crime (66.9\%) and strangers (66.2\%), and aesthetically beautiful (54.6\%). In contrast, a greater number of children were dissatisfied with the number of trees in their neighborhood (74.6\%), on the way to their school $(53.1 \%)$ and felt unsafe to play in the closest park without adult supervision (66.9\%). However, about half of the children had a feeling that they would get hurt while walking or cycling around $(50 \%)$. 
Table1. Summary statistics of socio-demographic variables included in this study

\begin{tabular}{|c|c|c|}
\hline Variable Name & Frequency $(\%)$ & Mean (SD) \\
\hline \multicolumn{3}{|l|}{ Child demography and individual factors } \\
\hline \multicolumn{3}{|l|}{ Gender } \\
\hline Male/ Female & $71(47.0) / 80(53.0)$ & - \\
\hline Age & - & $12.25(1.47)$ \\
\hline Number of siblings $\leq 18$ years old & - & $0.89(0.70)$ \\
\hline Number of car/motorbikes in family & - & $0.30(0.52)$ \\
\hline \multicolumn{3}{|l|}{ I have lots of friends in the neighborhood } \\
\hline $\begin{array}{ll} & \text { Yes/ No } \\
\end{array}$ & $105(69.5) / 46(30.5)$ & - \\
\hline \multicolumn{3}{|l|}{ I know lots of people in the neighborhood } \\
\hline $\begin{array}{ll} & \text { Yes/ No } \\
\end{array}$ & $112(74.2) / 39(25.8)$ & - \\
\hline \multicolumn{3}{|l|}{ Ownership of cycle } \\
\hline Yes/ No & $56(37.1) / 95(62.9)$ & - \\
\hline \multicolumn{3}{|l|}{ Case study area } \\
\hline Azimpur/ Madhubag/ Manikdi/ Uttara & $58(38.4) / 32(21.2) / 41(27.2) / 20(13.2)$ & - \\
\hline \multicolumn{3}{|l|}{ Family demography } \\
\hline Duration (in year) in current neighborhood & - & $8.97(7.44)$ \\
\hline \multicolumn{3}{|l|}{ Family income (taka) } \\
\hline $\begin{array}{l}\geq 25,000 / 26,000-50,000 / 51,000-1,00,000 / \\
1,00,000-2,00,000 / \leq 2,00,000\end{array}$ & $\begin{array}{r}45(29.8) / 64(43.0) / 34(22.5) / 6(4.0) / 1 \\
(0.7) \\
\end{array}$ & - \\
\hline \multicolumn{3}{|l|}{ Age of Mom (in year) } \\
\hline$<30 / 30-45 />45$ & $15(9.9) / 128.0(84.8) / 8.0(5.3)$ & - \\
\hline \multicolumn{3}{|l|}{ Age of Dad (in year) } \\
\hline$<30 / 30-45 />45$ & $0(0.0) / 87.0(57.60) / 64.0(42.40)$ & - \\
\hline \multicolumn{3}{|l|}{ Relation with participating child } \\
\hline Mother/ Father/ Other & $94(62.3) / 52(34.4) / 5(3.3)$ & - \\
\hline \multicolumn{3}{|l|}{ Child Perceived local environment } \\
\hline \multicolumn{3}{|l|}{ There is a lot of traffic in my neighborhood } \\
\hline $\begin{array}{rr}\text { Agree/ Disagree } \\
\end{array}$ & $48(36.9) / 82(63.1)$ & \\
\hline \multicolumn{3}{|l|}{ There is a lot of crime in my neighborhood } \\
\hline $\begin{array}{rr}\text { Agree/ Disagree } \\
\end{array}$ & $43(33.1) / 87(66.9)$ & \\
\hline \multicolumn{3}{|l|}{$\begin{array}{l}\text { I feel unsafe to play at the closest park without the } \\
\text { presence of any adult }\end{array}$} \\
\hline Agree/ Disagree & $43(33.1) / 87(66.9)$ & \\
\hline \multicolumn{3}{|l|}{$\begin{array}{l}\text { I might get hurt while walking or cycling due to } \\
\text { poor condition of roads }\end{array}$} \\
\hline $\begin{array}{r}\text { Agree/ Disagree } \\
\end{array}$ & $65(50.0) / 65(50.0)$ & \\
\hline \multicolumn{3}{|l|}{ I'm worried about strangers in my neighborhood } \\
\hline $\begin{array}{r}\text { Agree/ Disagree } \\
\end{array}$ & $44(33.8) / 86(66.2)$ & \\
\hline \multicolumn{3}{|l|}{$\begin{array}{l}\text { Roads of my neighborhood are aesthetically } \\
\text { beautiful }\end{array}$} \\
\hline $\begin{array}{l}\text { Agree/ Disagree } \\
\end{array}$ & $71(54.6) / 59(45.4)$ & \\
\hline \multicolumn{3}{|l|}{ There are enough trees in my neighborhood } \\
\hline Agree/ Disagree & $33(25.4) / 97(74.6)$ & \\
\hline \multicolumn{3}{|l|}{ There are enough trees on my way to school } \\
\hline Agree/ Disagree & $61(46.9) / 69(53.1)$ & \\
\hline
\end{tabular}




\subsection{Objective BE data collection}

Table 2 provides the description of the BE attributes (27 in total) comprised of geographic and routealong $(\mathrm{n}=23)$ factors, and syntactic attributes included in this study. The Objective geographic data were collected by employing a virtual walkability audit, and syntactic BE were derived from Depthmap.

\subsubsection{Walkability audit for route - along BE data}

For geographic route-along data collection, a virtual walkability audit was developed as a result of unavailable context and CIM-specific tool, based on the relevant local and global literature (BCA, 2008; Lee, Kim, Dowdy, Hoelscher, \& Ory, 2013; Sallis et al., 2015; Sharmin \& Kamruzzaman, 2017). One of the co-authors collected virtual walkability data using Google Street View as applied in Badland, Opit, Witten, Kearns, and Mavoa (2010) in December 2017. Data were collected for each segment on child-reported routes. In the case of unavailable Google Street View data of some segments ( $11 \%$ of the total of 681 segments), walkability data were collected from photographs and videos captured during the questionnaire survey.

\subsubsection{Depthmap for syntactic BE data}

The syntactic BE attributes were derived based on the space syntax concept and methodology in Depthmap, version X 0.50 software. Although the space syntax concept can be operationalised using two types of representations of urban spaces: axial and segment, this research used segment analysis. While axial representation analyses spatial network by the longest and least set of axial (straight) lines for illustration of small urban spaces, the segment representation uses segments formed by chopping the original axial lines at the junctions into smaller individual parts. Comparing segment and axial analyses, Hillier and Iida (2005) revealed that a segment representation is more precise and able to capture minor variations in direction change. Moreover, a recent meta-analysis on space syntax and pedestrian movement also found the better explanatory power of segment analysis than axial analysis (Sharmin \& Kamruzzaman, 2018a). Segment analysis is based on three types of distance concept: angular, topological and metric respectively defined as the sum of directional changes in degrees (least angle change), the number of directional changes required to reach a destination (fewest turns) and the sum of segment lengths in meter between an origin and a destination (Hillier \& Iida, 2005). This study applied angular method among three methods for the derivation of commonly used space syntax measures (i.e., integration, choice and connectivity) because comparative studies on these methods indicate that angular measures are the strongest predictor of travel behavior (Sharmin \& Kamruzzaman, 2018b; Xia, 2013).

A four-step process was followed to derive syntactic BE attributes. First, existing road network maps of the $3000 \mathrm{~m}$ buffer of each participant schools in ArcGIS, version 10.6 were prepared due to unavailability of up-to-date road network maps in local context. Given that children living in $1600 \mathrm{~m}$ buffer were only included in the study, the larger radius of analysis was considered because a larger radius minimises the edge effect of the spatial network in segment analysis as reported in Ratti (2004). Edge effect refers to the tendency of the edges of spatial system appearing disproportionately segregated than they might be in reality due to the fact that streets on the edge of the map are connected onward (Hillier, 2007). In addition, a larger radius allowed that children's discretionary trips were not bound to be between home and school. In this study, a minimum $1 \mathrm{~km}$ extra setback was secured from the furthest trip reported. Second, respective spatial axial maps were constructed in Depthmap based on prepared and imported ArcGIS road network maps. Third, segment maps were then constructed based on axial maps to run segment analysis. Fourth, segment-based 


\begin{tabular}{|c|c|c|c|c|c|c|c|c|c|c|c|c|c|c|c|c|c|c|}
\hline $\begin{array}{l}\widehat{\hat{\sigma}} \\
\text { है } \\
\text { हुँ }\end{array}$ & 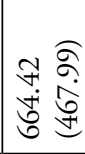 & 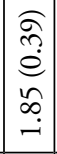 & 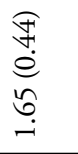 & 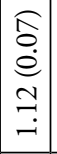 & 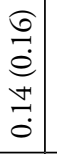 & $\begin{array}{l}\hat{\partial} \\
\dot{0} \\
\stackrel{0}{0} \\
\dot{0}\end{array}$ & 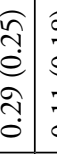 & $\begin{array}{l}\infty \\
\overrightarrow{0} \\
\stackrel{-}{0} \\
\vec{\Xi} \\
\overrightarrow{0}\end{array}$ & ' & 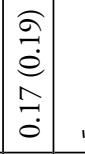 & . & $\begin{array}{l}\text { Oे } \\
\stackrel{+}{0} \\
\tilde{n} \\
\text { i }\end{array}$ & 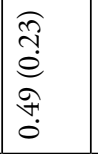 & & $\begin{array}{l}\overparen{\hat{~}} \\
\tilde{e} \\
\stackrel{0}{0} \\
\dot{n}\end{array}$ & 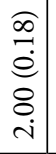 & $\begin{array}{l}\widehat{\widehat{o}} \\
\stackrel{0}{0} \\
\vdots \\
\dot{0}\end{array}$ & \\
\hline 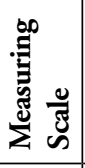 & 哇 & 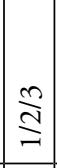 & $\stackrel{m}{\stackrel{m}{I}}$ & 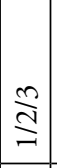 & $\stackrel{1}{=}$ & $\stackrel{9}{\triangleq}$ & $\stackrel{\ominus}{\varrho}$ & $\stackrel{\varrho}{=}$ & 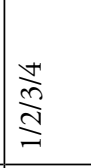 & 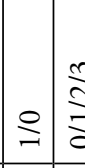 & 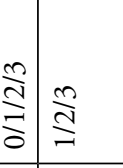 & $\stackrel{m}{\stackrel{m}{I}}$ & - & 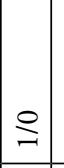 & 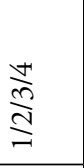 & 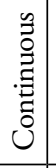 & $\stackrel{\varrho}{\leftrightharpoons}$ & $\stackrel{m}{s}$ \\
\hline 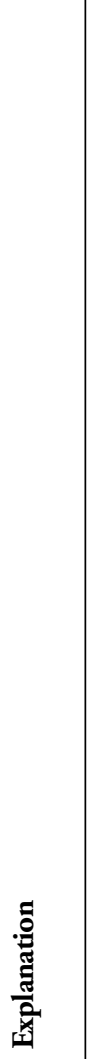 & 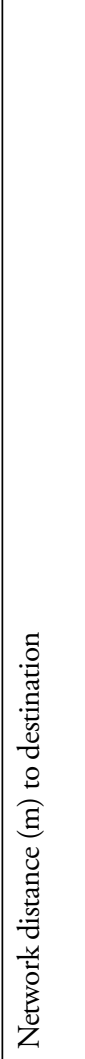 & 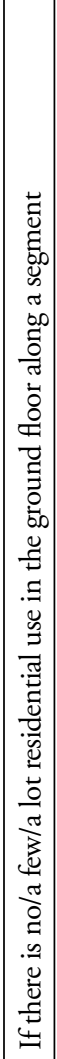 & 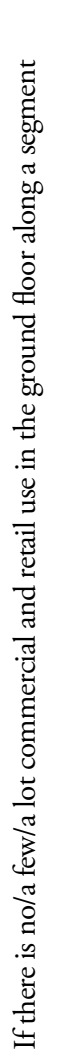 & 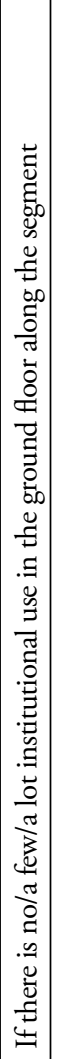 & 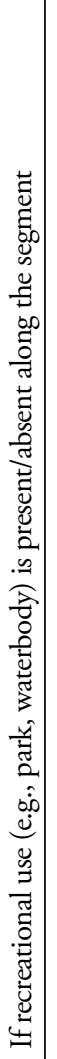 & 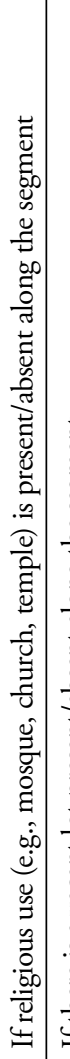 & 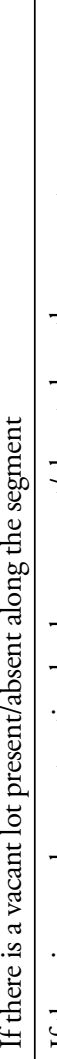 & 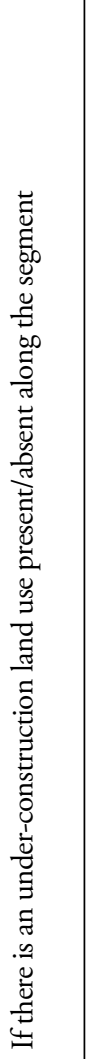 & 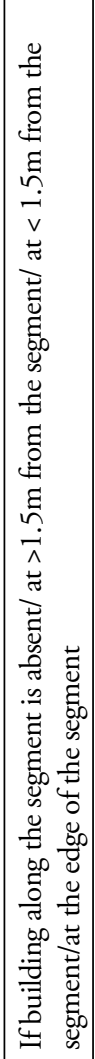 & 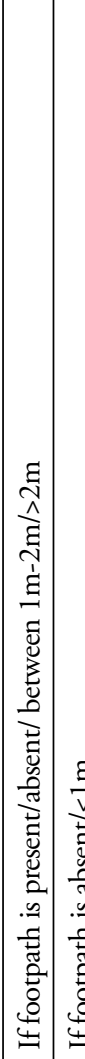 & 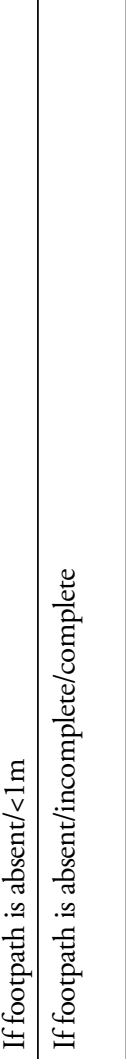 & 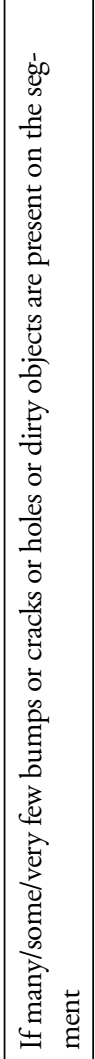 & 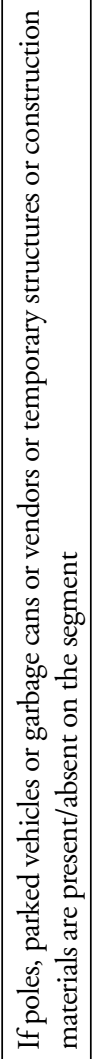 & 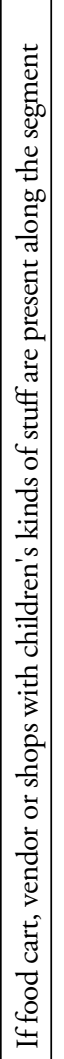 & 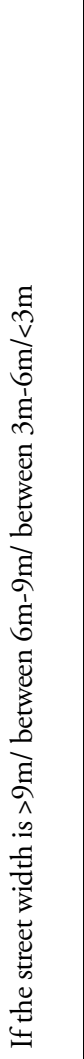 & 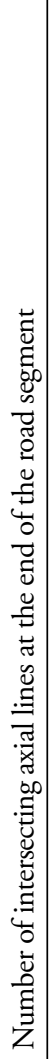 & 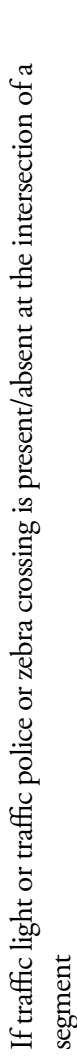 & 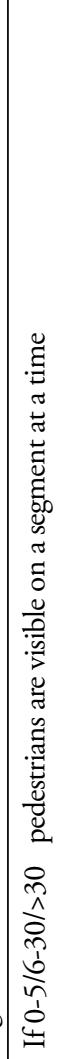 \\
\hline 离 & ' & 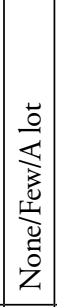 & 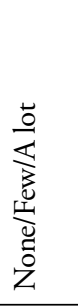 & 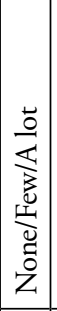 & $\sum_{\substack{3 \\
:}}^{Z}$ & 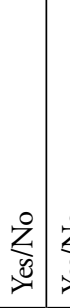 & 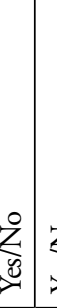 & $\sum_{\substack{x\\
}}$ & 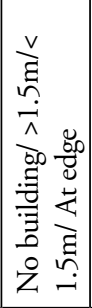 & 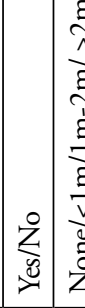 & 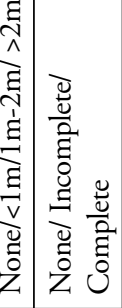 & 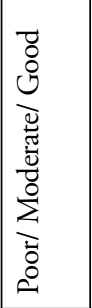 & 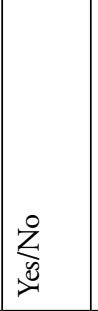 & 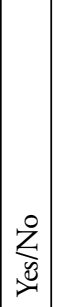 & 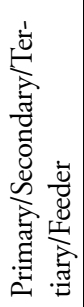 & & $\sum_{\substack{x\\
}}$ & 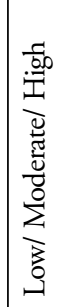 \\
\hline 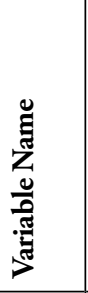 & 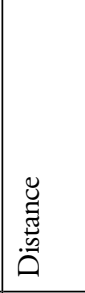 & 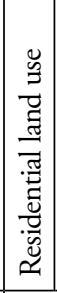 & 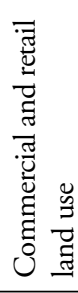 & 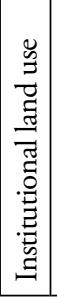 & 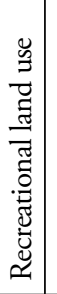 & 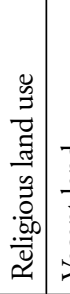 & . & 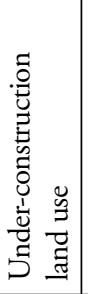 & 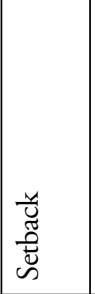 & 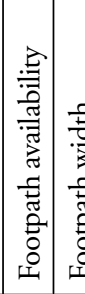 & 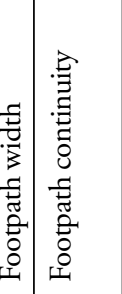 & 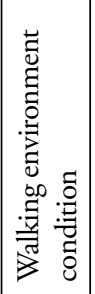 & 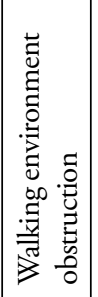 & 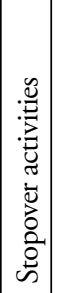 & 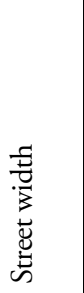 & 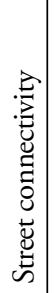 & 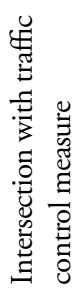 & 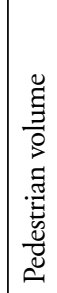 \\
\hline 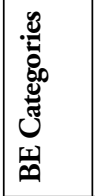 & 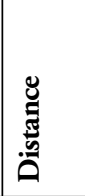 & 总 & & & & & & & & \multicolumn{5}{|c|}{ 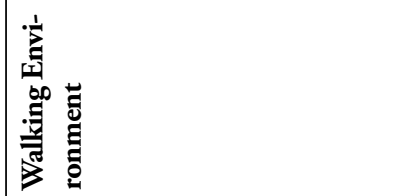 } & \multicolumn{4}{|l|}{ 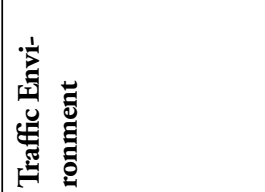 } \\
\hline 詻 & \multicolumn{18}{|c|}{ 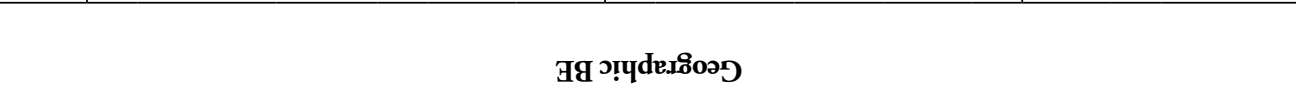 } \\
\hline
\end{tabular}




\begin{tabular}{|c|c|c|c|c|c|c|c|c|c|}
\hline 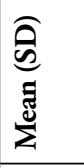 & 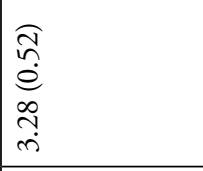 & 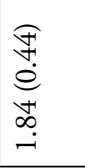 & 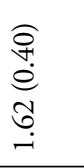 & ' & 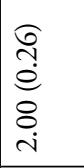 & 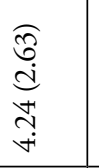 & 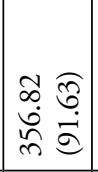 & 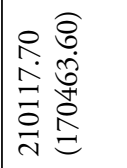 & 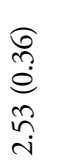 \\
\hline 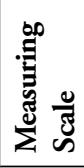 & 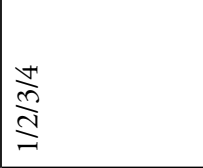 & $\stackrel{m}{\stackrel{m}{\beth}}$ & 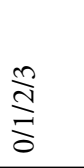 & $\stackrel{m}{\stackrel{m}{I}}$ & $\stackrel{m}{\stackrel{m}{=}}$ & 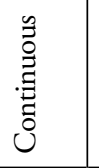 & 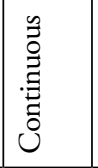 & 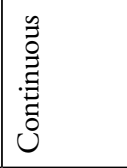 & 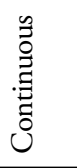 \\
\hline 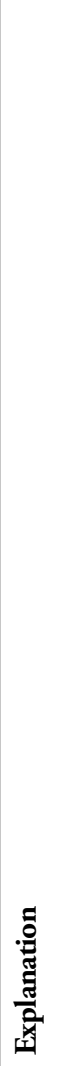 & 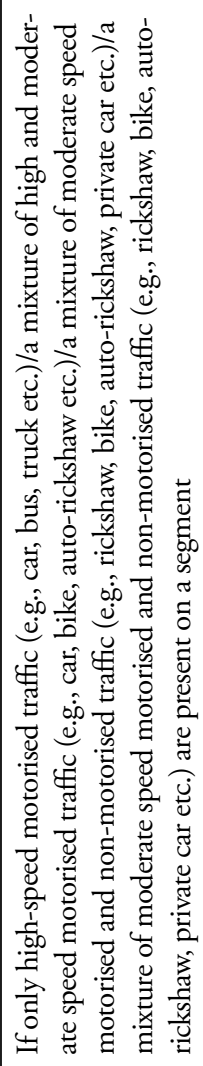 & 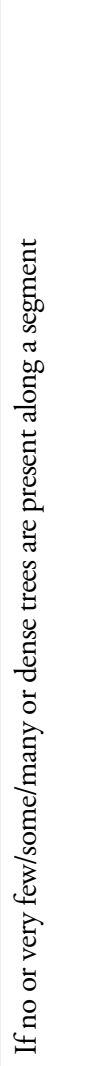 & 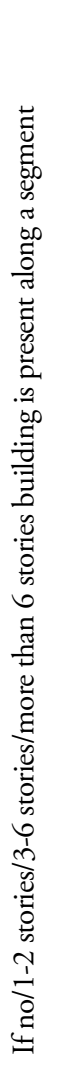 & 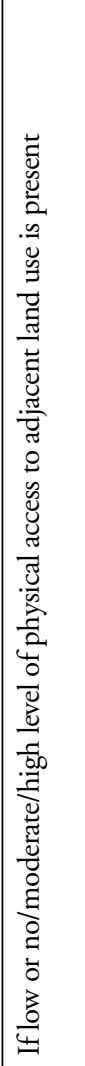 & 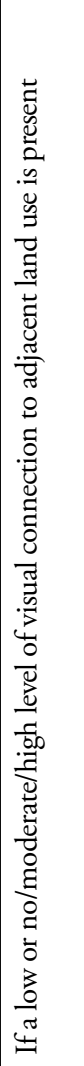 & 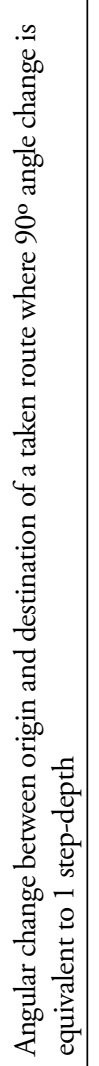 & 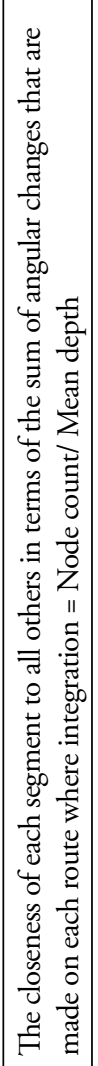 & 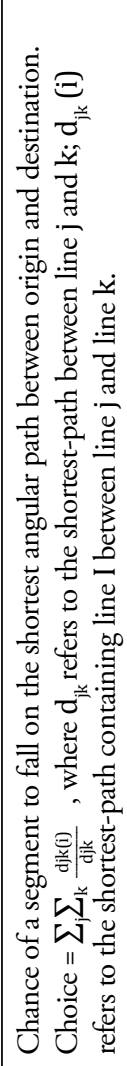 & 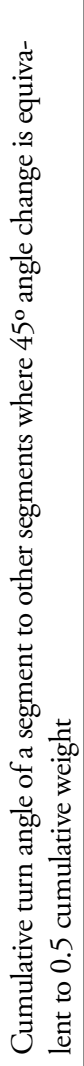 \\
\hline 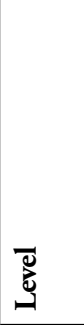 & 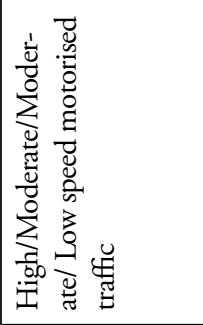 & 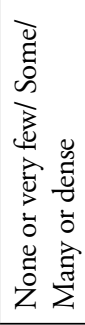 & 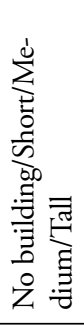 & 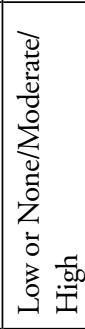 & 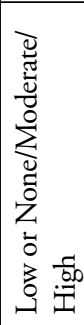 & ' & & & \\
\hline 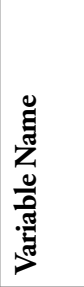 & 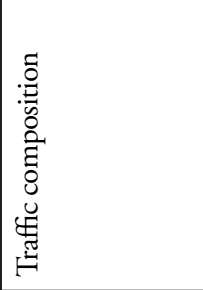 & 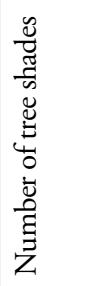 & 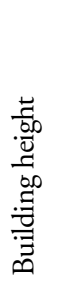 & 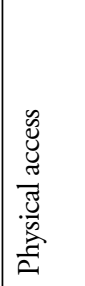 & 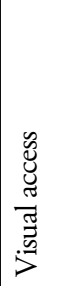 & 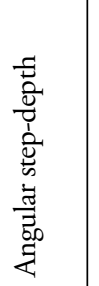 & 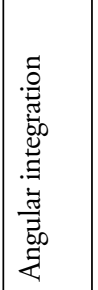 & 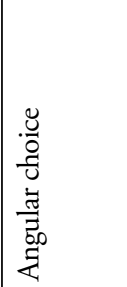 & 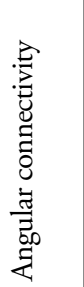 \\
\hline 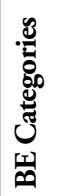 & 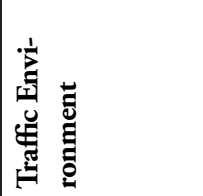 & \multicolumn{4}{|c|}{ 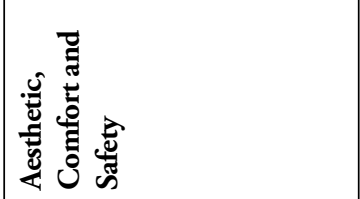 } & \multicolumn{4}{|c|}{ 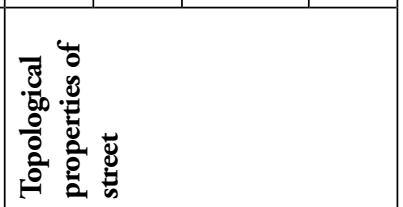 } \\
\hline & \multicolumn{5}{|c|}{ 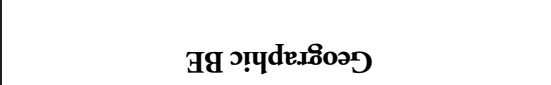 } & \multicolumn{4}{|c|}{ 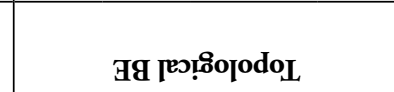 } \\
\hline
\end{tabular}




\subsection{Data entry, creation and processing}

\subsubsection{Digitizing visited destinations and routes}

Using ArcGIS application, all reported origins, destinations and taken routes of each participant were geocoded and digitized manually. The geographic dataset provided by the Capital Development Authority of Bangladesh was utilised in this study. This dataset included land use and street layout information of selected areas. Due to unavailability of up-to-date GIS data in some places, few streets and land uses were updated manually before digitizing reported routes.

\subsubsection{Measurement of TR}

Moore and Young (1978) proposed three levels of involvement for TR named "habitual," "frequented" and "occasional." Habitual-range includes close to home behavior, which begins to develop in early childhood. Frequented-range reflects the temporal flexibility of weekend and vacation periods and occasional-range relates to exotic destinations beyond the frequented range that are an experiential opportunity for some children. This study was particularly focussed on habitual TR during two days of the survey period. TR in this study was defined as the maximum distance children travelled to specific destinations without adult's supervision regardless of their mode of transportation.

In the travel diary, children reported the places they visited and accompaniment of each trip. A dichotomous measure of independent mobility was defined to indicate whether children travelled independently or not. A value of one was assigned for trips without adult accompaniment and a value of zero was assigned for trips with adult accompaniment. The independent trips were then separated and stratified into discretionary and nondiscretionary trips based on the destination type to calculate the TR. Discretionary trips included a wide range of destination including park/ playground, shop, religious centre (mosque/church/temple), friend's house, relative's house, library, club, café and cycling/walking those were optional for children to attend. On the other hand, nondiscretionary trips included school, coaching and other extra-curricular activities that were mandatory to attend regularly. As per the definition of TR, it expresses the furthest distance children travelled independently. TR was measured for three types of trips in this study: i) for all independent trips regardless of the type of destinations named as "overall TR;" ii) for discretionary independent trips only named as "discretionary TR;" and iii) for nondiscretionary trips only named as "nondiscretionary TR." At first, the independent range of each reported trip was calculated by network distance in ArcGIS. In most of the cases, students reported multiple trips ranging from two to four in two days of survey. By comparing multiple trip ranges reported by each student, the independent TR (furthest trip distance travelled independently) of each child was then selected for overall trips, discretionary and nondiscretionary trips. The overall, discretionary and nondiscretionary TRs were then $\log$ (natural) transformed to account for the nonlinear relationship between TR and explanatory variables.

\subsubsection{Measurement of route - along BE attributes}

Given that all BE data were collected for each segment, they were aggregated for each reported route. The segment values of syntactic measures and route-along BE were imported and assigned to respective route-segments in ArcGIS. In the next step, the values of respective segment level attributes were averaged based on the number of segments forming a route. Table 2 presents the summary statistics (mean and standard deviation) of all $\mathrm{BE}$ attributes for reported independent routes. 


\subsection{Statistical analysis}

Analyses were conducted using Stata/SE 15.1. Participants with complete and valid independent travel data ( $\mathrm{n}=130$ sample units, where 1 unit=1 child and 1 parent) were included in the analysis. Linear regression analyses were performed to examine how route-along $\mathrm{BE}$ attributes, perceived environment of children and socio-demography of children and parents were associated with TR. At first, a correlation analysis was conducted to check the correlations between distance and depth (Appendix 1). The result shows that distance and depth are correlated with a lower value. Then another correlation analysis was conducted to check the correlations among the $27 \mathrm{BE}$ variables including depth. Based on the results, six variables with a higher correlation value (value $>0.7$ ) (i.e., setback, footpath width, footpath continuity, stopover activities, pedestrian volume and physical access) were excluded from this study (Appendix 2). Regression analyses with remaining $21 \mathrm{BE}$ variables were then conducted in two-steps. First, a series of bivariate linear regression analyses examined cross-sectional associations between each explanatory variable (BE, socio-demographic and perceived environment variables) and the $\ln (\mathrm{TR})$ to overall, discretionary and nondiscretionary destinations separately. Second, significant variables $(\mathrm{p}<0.1)$ for overall, discretionary and nondiscretionary TR were entered into the respective adjusted multiple linear regression models. Using the fitted models, the marginal effects of significant explanatory variables were computed and reported in Table 4. Marginal effects represent the corresponding change in TR range for one unit change in a continuous variable or conditional change ( 0 to 1$)$ in an indicator variable, holding all other variables constant.

\section{Results: descriptive and statistical}

\subsection{TR to different destinations}

Among 466 total trips, 70\% of trips were independent. The reason for a large share of CIM trips in the context of this study compared to other contexts (Fagan-Watson et al., 2015) might be the result of two factors: 1) unlike previous studies that mainly focused on non-discretionary trips, this study captured both discretionary and non-discretionary trips over two days comprising of both weekdays and weekends; and 2) this study is one of the few studies that focused on a developing country context where motorised vehicle ownership has been historically low, and therefore, the children are less likely to be chauffeured by their parants.

A total of 130 independently mobile children made 327 trips to various local destinations, among which more than half of the trips $(52.9 \%)$ were discretionary trips. Discretionary trip number per child is 1.77 (173 trips made by 98 independent children) whereas it is 1.43 (154 trips made by 107 independent children) for nondiscretionary trips. Despite the dominance of the number of reported discretionary trips, more children were independently mobile to nondiscretionary destinations. Among 130 independently mobile children, 98 reported discretionary CIM and 107 reported nondiscretionary CIM. The nondiscretionary TR is found to be almost double in length than the discretionary TR. While the mean nondiscretionary TR was $664.14(\mathrm{SD}=44.04)$, the mean range for discretionary was $397.89 \mathrm{~m}(\mathrm{SD}=32.79)$. Despite a higher mean value of TRs, the minimum TR for discretionary trips was only $38.01 \mathrm{~m}$ compared to $110.33 \mathrm{~m}$ for nondiscretionary trips. A closer look at the stratified TR revealed that about $75 \%$ of children had a TR of discretionary trips ranged below $500 \mathrm{~m}$ whereas only $44 \%$ of children had TR of nondiscretionary trips ranged below $500 \mathrm{~m}$. The discretionary TR is very small $(<200 \mathrm{~m})$ for almost $11 \%$ of children ( $11 \%$ of trips), whereas only $2.8 \%$ of children had nondiscretionary TR ranged less than or equal to $200 \mathrm{~m}$. Figure 1 presents the TR of various discretionary and nondiscretionary destinations. It is quite clear that school was the furthest $(2103 \mathrm{~m})$ destination for 
nondiscretionary trips. After leisure walk/cycle, among the other discretionary destinations, park was the furthest $(908.61 \mathrm{~m})$ discretionary destination for children.

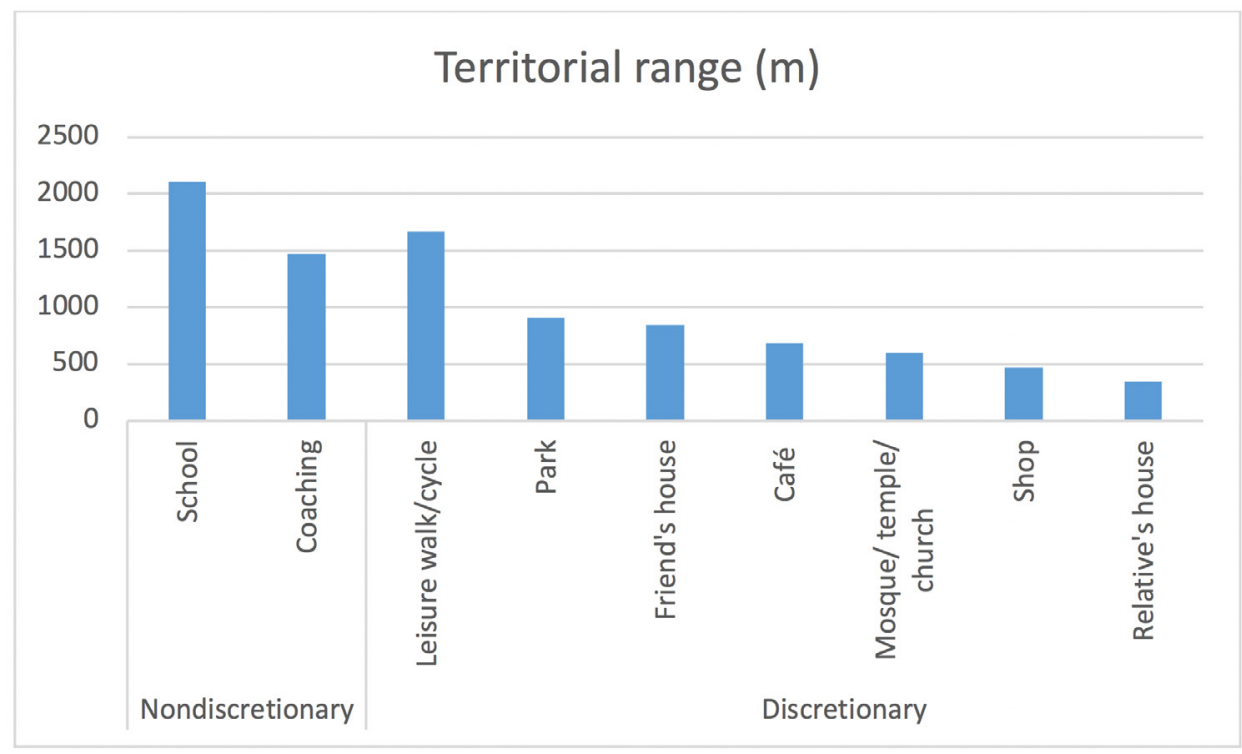

Figure 1. Territorial range of discretionary and nondiscretionary destinations

\subsection{Factors affecting overall, discretionary and nondiscretionary TR}

Table 3 presents the estimated parameters of the multiple linear regression models for overall, discretionary and nondiscretionary TR. The models indicate a reasonable level of fit with 55.8\%, 62.8\% and $50.8 \%$ explained variation in child's overall, discretionary and nondiscretionary TR. The F-tests, as reported in Table 3, suggest that each model has sufficient explanatory power compared to a null model. Table 4 presents the marginal effects of significant explanatory variables. It enables a better understanding of the corresponding change in TR for one unit change in a particular variable, holding all other variables constant. The findings from these models are discussed below:

\subsubsection{Effects of built-environment attributes on TR}

The TR of children is found to be highly associated with en-route BE attributes. The influence of BE predictors is different for overall, discretionary and nondiscretionary ranges due to the varying nature of these trips.

Among the four significant $\mathrm{BE}$ attributes for overall $\mathrm{TR}$, one belongs to syntactic $\mathrm{BE}$, and the rest of the $\mathrm{BE}$ factors belong to geographical $\mathrm{BE}$. The significant syntactic $\mathrm{BE}$ factor is angular stepdepth. On the other hand, the significant geographical BE factors are the presence of residential land use, walking environment condition and number of tree shades along the routes. Angular step-depth, walking environment condition and number of trees along the route have positive associations, and the residential land use has negative associations. While the quality of walking environment condition predicts the largest positive impact on overall TR, angular step-depth holds the smallest positive impact. In particular, overall TR increases by $318.14 \mathrm{~m}$ if the quality of walking environment condition increases by one unit from "poor" to "moderate" or from "moderate" to "good." Similarly, one unit increase in number of trees along the routes (from "none/few" to "some" or "some" to "many/dense") and angular 
step-depth ( 1 turn $/ 90^{\circ}$ change) respectively increase overall TR by $223.84 \mathrm{~m}$ and $65.92 \mathrm{~m}$. In contrast, the overall TR decreases by $138.91 \mathrm{~m}$ if residential land use increases by one level from "none" to "few" or "few" to "a lot."

For discretionary TR, angular step-depth, walking environment condition, and street connectivity are significant predictors. Unlike overall TR, all significant predictors show only positive influences on TR. The largest impact was reported by street connectivity factor while the smallest was by angular stepdepth. In other words, the discretionary TR extends by $293.14 \mathrm{~m}$ if the number of street connectivity increases by one unit and by $124.23 \mathrm{~m}$ if the walking environment condition of the route is better and free from bumps, holes and cracks and dirty objects. Similarly, a one unit increase of depth (or 1 turn equivalent to $90^{\circ}$ change of angular step-depth) results in $63.52 \mathrm{~m}$ extension of discretionary TR.

Among the three significant predictors for nondiscretionary TR, angular step-depth and condition of walking environment are positively associated, and the presence of commercial and retail land use is negatively associated with nondiscretionary TR. Walking environment condition has been found to have the largest impact and the angular step-depth has been found to have the smallest impact. For instance, when a route is free from bumps or cracks or holes or dirty objects, the nondiscretionary range experiences a rise of $312.21 \mathrm{~m}$. On the other hand, the presence of commercial and retail land use along the route causes the shrinkage of nondiscretionary range by $82.95 \mathrm{~m}$.

\subsubsection{Effects of perception and socio-demography on TR}

As reported in Table 3, three perceptual factors of children are found to be significant for TR. The significant perceptual factors are: the level of crime in the neighborhood, the number of trees in the neighborhood and the number of trees on the way to school. While concern about the level of crime in the neighborhood is associated with overall TR, satisfaction with the number of trees in the neighborhood and number of trees on the way to school are respectively associated with discretionary and nondiscretionary TRs. Concern about the level of the crime in the neighborhood has a negative impact on TR whereas satisfaction with the number of trees has a positive impact on TR. positively associated, and commercial and retail land use is negatively associated with nondiscretionary TR, As presented in Table 4 , child's fear about the crime is one of them which reduces the overall TR by $30.93 \mathrm{~m}$. On the contrary, children's satisfaction with the number of trees in the neighborhood increases the nondiscretionary TR by $86.22 \mathrm{~m}$. Similarly, if children are happy with the amount of tress on the way to school, their nondiscretionary expands TR by $41.81 \mathrm{~m}$.

Girls have a somewhat smaller size of TR than boys irrespective of the type of TR. The TR difference is smaller for discretionary activities and larger for nondiscretionary activities. Girls in comparison to boys travel $58.15 \mathrm{~m}, 23.94 \mathrm{~m}$ and $40.83 \mathrm{~m}$ less for overall, discretionary and nondiscretionary trips respectively. The socio-demographic factors of parents show differential effects on children's independent TR. Children from a family where father's highest education is masters reported $66.50 \mathrm{~m}$ increase of discretionary TR. On the other hand, with the increase of dad's age, TR shrinks, as children reported to travel $74.91 \mathrm{~m}$ less to nondiscretionary destinations when the father is $>45$ years old. 


\begin{tabular}{|c|c|c|c|c|c|c|c|c|c|c|c|c|c|c|c|c|c|c|c|c|}
\hline \multirow{6}{*}{ 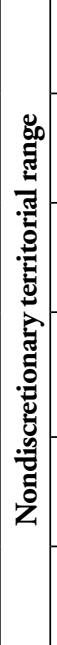 } & 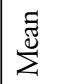 & $\stackrel{\triangleq}{\overrightarrow{+i}}$ & & $\underset{-}{\hat{\sigma}}$ & & $\begin{array}{l}\stackrel{\sim}{n} \\
i\end{array}$ & & $\tilde{n}$ & $\underset{\sim}{\stackrel{7}{i}}$ & ' & & ' & $\stackrel{n}{\hat{0}}$ & ' & & & & & & \\
\hline & ڤ̊ & {$\left[\begin{array}{cc}\hat{\hat{o}} & \stackrel{7}{0} \\
0 & 0\end{array}\right.$} & ' & 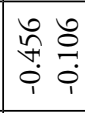 & & 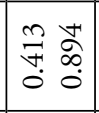 & ' & 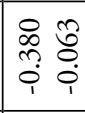 & 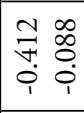 & ' & ' & ' & $\mid \begin{array}{ll} & \infty \\
0 & 0 \\
0 & 0 \\
0 & 0 \\
0\end{array}$ & 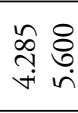 & & & & & & \\
\hline & $\frac{\bar{E}}{\hat{\Lambda}}$ & $\ddot{\circ}$ & & $\begin{array}{l}\widetilde{\sigma} \\
\stackrel{0}{0}\end{array}$ & & $\vec{\Xi}$ & & 客 & 离 & ' & & ' & 息 & $\overrightarrow{\tilde{o}}$ & $\underset{\substack{f\\
}}{0}$ & $\overrightarrow{0}$ & $\left|\begin{array}{l}\infty \\
0 \\
0 \\
0\end{array}\right|$ & $\begin{array}{c}\infty \\
\stackrel{\infty}{\ddagger} \\
0\end{array}$ & $\wedge$ & $\hat{O}$ \\
\hline & - & 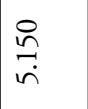 & & 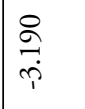 & & ஓे & & 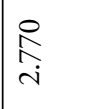 & $\begin{array}{l}8 \\
\dot{0} \\
\dot{1}\end{array}$ & ' & 1 & ' & 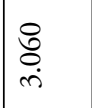 & $\begin{array}{l}\stackrel{\Xi}{\Omega} \\
\stackrel{-}{-}\end{array}$ & & & & & & \\
\hline & 㝴苞 & $\vec{\partial}$ & & $\begin{array}{l}\infty \\
\infty \\
0 \\
0\end{array}$ & & $\vec{\beth}$ & 1 & $\begin{array}{l}0 \\
\stackrel{0}{0} \\
0\end{array}$ & $\mid \begin{array}{l}0 \\
\stackrel{0}{0} \\
\stackrel{0}{0}\end{array}$ & ' & ' & ' & $\vec{\infty}$ & $\overrightarrow{\tilde{\sigma}}$ & & & & & & \\
\hline & ப் & ڤ్ & ' & $\begin{array}{l}\overrightarrow{0} \\
\stackrel{\sim}{i} \\
\stackrel{i}{i}\end{array}$ & ' & 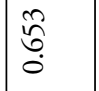 & . & $\begin{array}{l}\overrightarrow{\widetilde{N}} \\
\stackrel{i}{i}\end{array}$ & $\begin{array}{l}0 \\
\\
i \\
i\end{array}$ & ' & 1 & ' & $\underset{\stackrel{f}{+}}{\stackrel{0}{0}}$ & $\underset{\stackrel{F}{\forall}}{\stackrel{7}{+}}$ & & & & & & \\
\hline \multirow{6}{*}{ 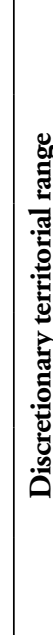 } & $\sum^{\bar{E}}$ & $\vec{\infty}$ & , & ' & $\widehat{\varrho}$ & ते & I & के & ' & ஸे & $\vec{\infty}$ & 1 & ' & ' & & & & & & \\
\hline & $\stackrel{\circ}{\alpha} 0$ & 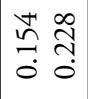 & ' & ' & 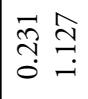 & 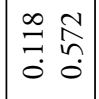 & ' & 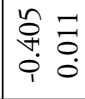 & ' & 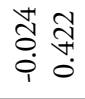 & . & $\begin{array}{ll}\widetilde{r} & \mathbb{N} \\
0 & 0 \\
0 & 0\end{array}$ & ' & 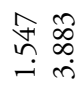 & & & & & & \\
\hline & $\frac{\bar{\sigma}}{\hat{\lambda}}$ & $\ddot{8}$ & ' & ' & $\begin{array}{l}n \\
8 \\
0\end{array}$ & $\begin{array}{l}0 \\
\delta \\
0\end{array}$ & ' & $\begin{array}{l}n \\
\ddot{0} \\
0 \\
0\end{array}$ & . & $\begin{array}{l}0 \\
0 \\
0 \\
0\end{array}$ & . & ڤ̊ & . & $\begin{array}{l}8 \\
\vdots \\
0\end{array}$ & $\mid \begin{array}{l}n \\
0 \\
+ \\
0 \\
0\end{array}$ & 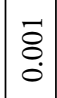 & 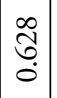 & $\mid \begin{array}{l}2 \\
0 \\
0 \\
0\end{array}$ & $\curvearrowright$ & $\stackrel{\infty}{\sim}$ \\
\hline & - & $\begin{array}{l}8 \\
0 \\
0\end{array}$ & ' & ' & $\vec{\sigma}$ & $\stackrel{0}{0}$ & I & $\begin{array}{l}\infty \\
\infty \\
\stackrel{0}{+} \\
\rightarrow-1\end{array}$ & ' & $\stackrel{R}{\stackrel{R}{i}}$ & ' & $\begin{array}{l}\stackrel{8}{0} \\
\ddot{n} \\
\dot{n}\end{array}$ & ' & $\begin{array}{l}\stackrel{\text { ్ָర }}{+} \\
\stackrel{+}{ }\end{array}$ & & & & & & \\
\hline & के & 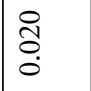 & ' & ' & $\begin{array}{l}\text { సิ } \\
\text { กิ }\end{array}$ & $\stackrel{\varpi}{\exists}$ & ' & $\begin{array}{l}\stackrel{n}{0} \\
\vdots \\
0\end{array}$ & ' & $\stackrel{7}{\exists}$ & ' & 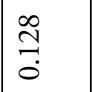 & ' & \begin{tabular}{l}
$\infty$ \\
$\infty$ \\
\hdashline \\
0
\end{tabular} & & & & & & \\
\hline & üं & $\vec{\sigma}$ & , & . & $\stackrel{0}{0}$ & 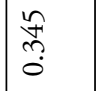 & ' & $\stackrel{\hat{a}}{\stackrel{0}{i}}$ & I & $\stackrel{2}{\overrightarrow{0}}$ & ' & $\stackrel{\infty}{\underset{\sigma}{*}}$ & & 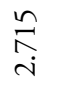 & & & & & & \\
\hline \multirow{6}{*}{ 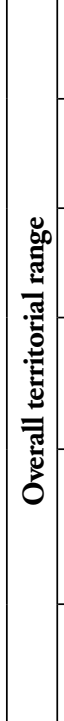 } & 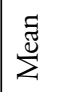 & $\underset{⿱}{\forall}$ & $\stackrel{n}{\infty}$ & ' & & $\widetilde{\sim}$ & $\stackrel{\infty}{\mathscr{C}}$ & 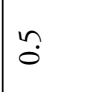 & ' & , & $\tilde{n}$ & ' & ' & ' & & & & & & \\
\hline & $\stackrel{\circ}{\alpha} 0$ & 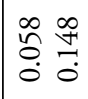 & 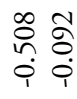 & ' & 1 & 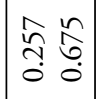 & 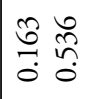 & 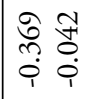 & ' & . & $\mid \begin{array}{cc}n & n \\
\text { N. } & \vdots \\
i & 0 \\
1 & 0\end{array}$ & ' & ' & 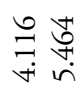 & & & & & & \\
\hline & $\frac{\bar{\Delta}}{\hat{A}}$ & $\overrightarrow{0}$ & 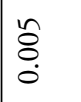 & . & & $\overrightarrow{8}$ & $\overline{8}$ & $\stackrel{H}{a}$ & & , & $\hat{n}$ & ' & & $\overrightarrow{8}$ & 悉 & $\overrightarrow{8}$ & $\mid \begin{array}{l}\infty \\
\tilde{n} \\
\hat{0}\end{array}$ & $\mid \begin{array}{l}0 \\
\tilde{n} \\
\hat{\delta}\end{array}$ & $\wedge$ & $\stackrel{\infty}{n}$ \\
\hline & + & ڤ̊. & 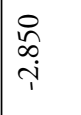 & ' & & 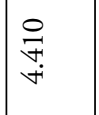 & 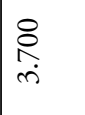 & 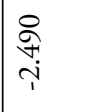 & , & ' & 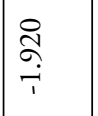 & ' & & $\begin{array}{l}\stackrel{R}{0} \\
\stackrel{0}{ \pm}\end{array}$ & & & & & & \\
\hline & $\begin{array}{l}\dot{1} \\
\text { ज्ञ } \\
\text { 离 }\end{array}$ & $\stackrel{0}{0}$ & $\begin{array}{l}n \\
\stackrel{\sigma}{0} \\
0\end{array}$ & ' & & $\frac{8}{0}$ & $\stackrel{\Delta}{0}$ & $\begin{array}{l}\stackrel{\partial}{0} \\
\stackrel{0}{0}\end{array}$ & & ' & $\begin{array}{l}a \\
\stackrel{0}{0} \\
\stackrel{0}{0}\end{array}$ & ' & & 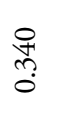 & & & & & & \\
\hline & üّ & $\stackrel{0}{=}$ & $\begin{array}{l}8 \\
\text { on } \\
i\end{array}$ & ' & & \begin{tabular}{l}
$l$ \\
\multirow{0}{0}{} \\
\multirow{0}{*}{}
\end{tabular} & ๙े? & 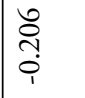 & & , & $\stackrel{2}{\stackrel{9}{9}}$ & ' & & $\underset{\forall}{\stackrel{\overbrace{}}{ף}}$ & & & & & & \\
\hline \multicolumn{2}{|c|}{ 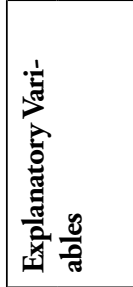 } & 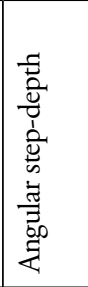 & 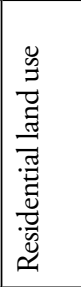 & 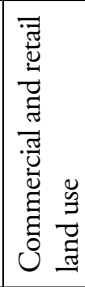 & 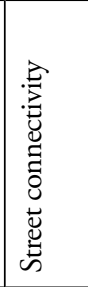 & 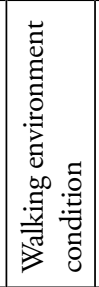 & 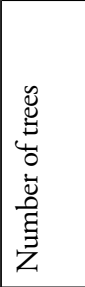 & 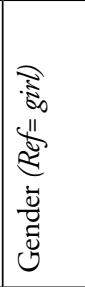 & 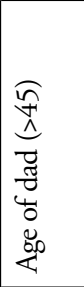 & 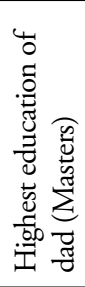 & 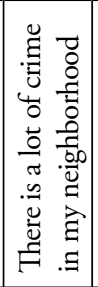 & 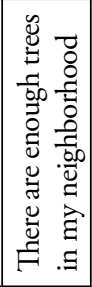 & 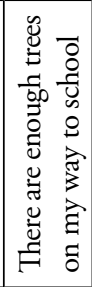 & $\tilde{0}$ & 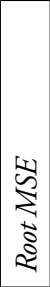 & $\begin{array}{l}1 \\
\hat{2} \\
\hat{2}\end{array}$ & 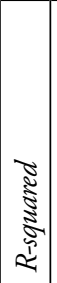 & 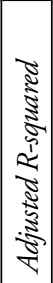 & \multicolumn{2}{|c|}{$z$} \\
\hline
\end{tabular}


Table 4. The marginal effects of significant explanatory variables

\begin{tabular}{|c|c|c|c|c|}
\hline $\begin{array}{l}\text { Explanatory } \\
\text { Variables }\end{array}$ & Calculation of TR decrease/increase & $\begin{array}{l}\text { Overall ter- } \\
\text { ritorial range } \\
\text { (TR) }\end{array}$ & $\begin{array}{l}\text { Discretion- } \\
\text { ary territorial } \\
\text { range }(T R)\end{array}$ & $\begin{array}{l}\text { Nondiscre- } \\
\text { tionary ter- } \\
\text { ritorial range } \\
\text { (TR) }\end{array}$ \\
\hline & $\ln (T R)$ at the mean of all variables & 6.28 & 5.71 & 5.83 \\
\hline & TR at the mean of all variables & 535.95 & 301.67 & 338.70 \\
\hline \multirow{3}{*}{$\begin{array}{l}\text { Angular step- } \\
\text { depth }\end{array}$} & $\ln (\mathrm{TR})$ for one-unit increase in step-depth & 6.40 & 5.90 & 5.92 \\
\hline & TR for one-unit increase in step-depth & 601.87 & 365.19 & 372.66 \\
\hline & TR increase for one-unit increase in step-depth & 65.92 & 63.52 & 33.95 \\
\hline \multirow{3}{*}{$\begin{array}{l}\text { Residential land } \\
\text { use }\end{array}$} & $\ln (\mathrm{TR})$ for one unit increase in residential land use & 5.98 & - & - \\
\hline & TR for one unit increase in residential land use & 397.04 & - & - \\
\hline & $\begin{array}{l}\text { TR decreases for one unit increase in residential } \\
\text { land use }\end{array}$ & -138.91 & - & - \\
\hline \multirow[t]{3}{*}{$\begin{array}{l}\text { Commercial and } \\
\text { retail land use }\end{array}$} & $\begin{array}{l}\ln (\mathrm{TR}) \text { for one unit increase in Commercial and } \\
\text { retail land use }\end{array}$ & - & - & 5.54 \\
\hline & TR for one unit increase in residential land use & - & - & 255.75 \\
\hline & $\begin{array}{l}\text { TR decreases for one unit increase in residential } \\
\text { land use }\end{array}$ & - & - & -82.95 \\
\hline \multirow{3}{*}{$\begin{array}{l}\text { Walking environ- } \\
\text { ment condition }\end{array}$} & $\ln (\mathrm{TR})$ for one unit increase in Footpath condition & 6.75 & 6.05 & 6.48 \\
\hline & TR for one unit increase in Footpath condition & 854.09 & 425.90 & 650.91 \\
\hline & $\begin{array}{l}\text { TR increase for one unit increase in Footpath } \\
\text { condition }\end{array}$ & 318.14 & 124.23 & 312.21 \\
\hline \multirow[t]{3}{*}{ Street connectivity } & $\ln (\mathrm{TR})$ for one unit increase in Street connectivity & - & 6.39 & - \\
\hline & TR for one unit increase in Street connectivity & - & 594.81 & - \\
\hline & $\begin{array}{l}\text { TR increase for one unit increase in Street con- } \\
\text { nectivity }\end{array}$ & - & 293.14 & - \\
\hline \multirow[t]{3}{*}{ Number of trees } & $\ln (\mathrm{TR})$ for one unit increase in Number of trees & 6.63 & - & - \\
\hline & TR for one unit increase in Number of trees & 759.79 & - & - \\
\hline & $\begin{array}{l}\text { TR increase for one unit increase in Number of } \\
\text { trees }\end{array}$ & 223.84 & - & - \\
\hline \multirow[t]{3}{*}{ Gender $($ Ref $=$ girl $)$} & $\ln (\mathrm{TR})$ for girls & 6.39 & 5.79 & 5.94 \\
\hline & TR for girls & 594.10 & 325.62 & 379.53 \\
\hline & TR decrease for girls & -58.15 & -23.94 & -40.83 \\
\hline \multirow[t]{3}{*}{ Age of dad $(>45)$} & $\begin{array}{l}\ln (\mathrm{TR}) \text { for one unit increase in Dad's highest } \\
\text { education }\end{array}$ & - & - & 5.58 \\
\hline & TR for one unit increase in Dad's highest education & - & - & 263.80 \\
\hline & $\begin{array}{l}\text { TR increase for one unit increase in Dad's highest } \\
\text { education }\end{array}$ & - & - & -74.91 \\
\hline \multirow[t]{3}{*}{$\begin{array}{l}\text { Highest education } \\
\text { of dad (Masters) }\end{array}$} & $\begin{array}{l}\ln (\mathrm{TR}) \text { for one unit increase in Dad's highest } \\
\text { education }\end{array}$ & - & 5.91 & - \\
\hline & TR for one unit increase in Dad's highest education & - & 368.17 & - \\
\hline & $\begin{array}{l}\text { TR increase for one unit increase in Dad's highest } \\
\text { education }\end{array}$ & - & 66.50 & - \\
\hline
\end{tabular}




\begin{tabular}{|c|c|c|c|c|}
\hline $\begin{array}{l}\text { Explanatory } \\
\text { Variables }\end{array}$ & Calculation of TR decrease/increase & $\begin{array}{l}\text { Overall ter- } \\
\text { ritorial range } \\
\quad(\mathrm{TR})\end{array}$ & $\begin{array}{l}\text { Discretion- } \\
\text { ary territorial } \\
\text { range }(T R)\end{array}$ & $\begin{array}{l}\text { Nondiscre- } \\
\text { tionary ter- } \\
\text { ritorial range } \\
\text { (TR) }\end{array}$ \\
\hline \multirow{3}{*}{$\begin{array}{l}\text { There is a lot } \\
\text { of crime in my } \\
\text { neighborhood }\end{array}$} & $\ln (\mathrm{TR})$ for Concern about crime in neighborhood & 6.34 & - & - \\
\hline & TR for Concern about crime in neighborhood & 566.88 & - & - \\
\hline & $\begin{array}{l}\text { TR decrease for Concern about crime in neighbor- } \\
\text { hood }\end{array}$ & -30.93 & - & - \\
\hline \multirow{3}{*}{$\begin{array}{l}\text { There are enough } \\
\text { trees in my neigh- } \\
\text { borhood }\end{array}$} & $\begin{array}{l}\ln (\mathrm{TR}) \text { for Satisfaction about the amount of neigh- } \\
\text { borhood trees }\end{array}$ & - & 5.37 & - \\
\hline & $\begin{array}{l}\text { TR for Satisfaction about the amount of neighbor- } \\
\text { hood trees }\end{array}$ & - & 215.46 & - \\
\hline & $\begin{array}{l}\text { TR decrease for Satisfaction about the amount of } \\
\text { neighborhood trees }\end{array}$ & - & 86.22 & - \\
\hline \multirow{3}{*}{$\begin{array}{l}\text { There are enough } \\
\text { trees on my way } \\
\text { to school }\end{array}$} & $\begin{array}{l}\ln (\mathrm{TR}) \text { for Satisfaction about the amount of neigh- } \\
\text { borhood trees }\end{array}$ & - & - & 5.69 \\
\hline & $\begin{array}{l}\text { TR for Satisfaction about the amount of neighbor- } \\
\text { hood trees }\end{array}$ & - & - & 296.89 \\
\hline & $\begin{array}{l}\text { TR decrease for Satisfaction about the amount of } \\
\text { neighborhood trees }\end{array}$ & - & - & 41.81 \\
\hline
\end{tabular}

\section{Discussion}

This paper investigates the TR of children to discretionary and nondiscretionary destinations, and how they differ from each other and from the overall $\mathrm{TR}$ in relation to the $\mathrm{BE}$ and socio-demographic factors. The following sub-sections discuss the key findings of this study.

\subsection{Nondiscretionary TR is greater than discretionary TR}

This research reveals that children travelling to discretionary and nondiscretionary destinations have a substantial difference in their TR. Although the overall TR is $664.42 \mathrm{~m}$, the stratified range shows the dominance of nondiscretionary TR over the discretionary in this study. Nondiscretionary TR $(664.14 \mathrm{~m})$ is almost $40 \%$ greater than the discretionary range, despite the larger number of discretionary trips reported. Surprisingly, $11 \%$ of children never ventured more than $200 \mathrm{~m}$ from their home to discretionary places. A greater number of discretionary trips within a much smaller range $(200 \mathrm{~m})$ indicates that: 1$)$ a higher number/proportion of independent trips do not necessarily mean a larger TR; and 2) when a greater diversity exists in discretionary destinations within a short distance, children might feel discouraged to move further. This might be the reason for the large difference between discretionary and nondiscretionary TR found in a dense urban area.

Notably, the mean TRs found in this study are shorter (one-third approx.) than the traditional walkable range/distance for an adolescent child $(1600 \mathrm{~m}$ ) (Carver et al., 2014; Timperio, Crawford, Telford, \& Salmon, 2004), although TR was measured in this study included both active (walking and cycling) and inactive modes of transport. The TR could have gone beyond $1600 \mathrm{~m}$ buffer as inactive modes were included, but it didn't. This reflects on findings that children of 10-12 years old partially use their 1600m network buffer (only 25\%) (K. Villanueva, Giles-Corti, Bulsara, McCormack, et al., 2012). The overall, discretionary and nondiscretionary TRs differ from the findings of Van Vliet (1983), who found that TR for the majority of participants is beyond $800 \mathrm{~m}$. However, The TRs partially align with Shaw et al. (2013) who showed that $35 \%$ of children independently travel up to $500 \mathrm{~m}$ from home. 
These findings highlight the importance of interventions aiming at range extension to all kind of destinations, especially for discretionary activities which children take more frequently.

\subsection{Built environment impacts nondiscretionary and discretionary TR in a different way}

This study found different sets of promoters and barriers to discretionary and nondiscretionary TR. The key variations are discussed on three levels. First, the syntactic BE attribute (i.e., angular step-depth) has a significant positive association with both discretionary and nondiscretionary TRs. However, step-depth has a larger control on discretionary TR than nondiscretionary TR. The positive associations of angular step-depth with both types of TRs confirms that routes with a greater number of turns or step-depth can encourage children to travel extra mileage independently irrespective of the type of destination. This is in contrast with Zdrahalova and Boumova (2016) who found that visually non-straight routes can create a safety concern, and therefore, discourages children from travelling to school independently. This could be attributed to the urban quality of streets that makes some streets preferable, more exciting and subject to new surprises for what children are ready to choose a route with more directional changes thus extend their independent TR. In doing that, children also trade-off the perception of increased distance to destination that is correlated with increased directional change as reported in Jansen-Osmann and Wiedenbauer (2004). The result also shows that to some extent visual distance (depth) and metric distance as conceptualized by respective topological and geographic school of thoughts to measure distance between origin-destination have a similar impact on TR. The practical implication of these results would be the provision of destinations with routes that have higher number of turns of step-depth (visual distance) for TR extension.

Second, land use (i.e., commercial and retail land use) largely correlates with nondiscretionary TR, whereas traffic environment (i.e., street connectivity) largely correlates with discretionary TR. The walking environment affects both types of TRs. Since the nondiscretionary destinations are not located in close proximity to home and the nature of the trips are mandatory, factors that provide a sense of comfort, safety and ease of movement encourages children to increase TR. On the other hand, for discretionary trips that are in general short-ranged from home, land use is not important to children; instead; street characteristics and ease of movement are important determinants of TR.

The negative impact of commercial and retail along the route on nondiscretionary TR, as found in this study, suggests that the presence of commercial and retail activities and the growth of crowds along with these activities as a by-product of street characteristics hinders children's TR to nondiscretionary destinations. This finding aligns with previous studies (Can \& Heath, 2016; Lopes, Cordovil, \& Neto, 2014) who claimed that an increased amount of crowd can increase the fear of stranger danger among children. This can consequently discourage children to choose crowded streets with a larger number of strangers to travel extra mileage while moving independently to nondiscretionary destinations such as school. The fear of stranger is a significant factor for both parents and children when it came to children's outdoor activities (Alparone \& Pacilli, 2012; Bwire, 2011; De Meester, Van Dyck, De Bourdeaudhuij, \& Cardon, 2014; Lopes et al., 2014; Mitra, Faulkner, Buliung, \& Stone, 2014). Furthermore, en-route commercial and retail activities often attract a high volume of traffic. This, in turn, increases the perception of lack of safety and security among children and therefore discourages children from travelling the extra mileage along the routes. Traffic safety is the biggest concern for both children and parents for CIM regardless of the age and gender of children (Carver et al., 2014; Collins \& Kearns, 2001; Hillman et al., 1990; Tranter \& Doyle, 1996; Villanueva, Giles-Corti, Bulsara, Timperio, et al., 2012; Villanueva et al., 2013). The mandatory nature and the larger TR of nondiscretionary trips might influence children to choose a safer route which has less amount of commercial and retail activities, less crowd and less traffic volume. 
Residential land use is a unique en-route BE feature that is only found to correlate to overall TR. The negative impact of the en-route residential land on overall TR, as found in this study, contrasts with the general assumption that higher density and compact structure in a neighborhood reduces the distance by increasing proximity, thereby, increases the extent of CIM (Prezza et al., 2001). The contrasting finding could be resulted from a difference in CIM measurement, as the reported study measured CIM by mobility licence. The negative impact of residential land use, however, is in line with a recent study which found that higher residential footprint in a neighborhood hinders CIM when measured by independent time spent outside (Loebach \& Gilliland, 2014). This negative correlation might be because of the dominance of en-route residential land that restricts and effectively reduces the establishment of other destinations of children's interests, which consequently limits children from moving further. These findings indicate that policymakers and $\mathrm{BE}$ professionals need to make a cautious balance between distribution of various land uses along the routes, and when aiming for TR extension, understanding the type of TR of children is important.

The positive impact of street connectivity, which measures how connected a street segment is with all other segments in the street network confirms that children prefer routes correspond to the higher connectivity value of segments while independently moving to different discretionary destinations. This behavior of children aligns with previous studies (Monsur, Mansur, \& Islam, 2017; Villanueva, GilesCorti, Bulsara, Timperio, et al., 2012; Villanueva et al., 2013) that well-connected streets have a positive influence on CIM. The findings, however, contrast that the previous findings on this topic that dead-end street (opposite to connected street) caters less traffic volume consequently increases CIM (Monsur et al., 2017; Sharmin \& Kamruzzaman, 2017; Sharmin, Kamruzzaman, \& Haque, 2020). The finding also differs from the general assumption that connected streets are characterised by having a higher potential of traffic movement and speed, and therefore, increase traffic danger among children (Page et al., 2010). A possible explanation might be that previous CIM studies mostly measured CIM by the likelihood of making a trip. TR is different from whether someone is making a CIM trip or not. Street connectivity can be an influence to decide whether a CIM trip to be made or not. Once the decision is taken, children are no longer afraid of facing the challenges of connected routes, instead, they enjoy roaming around connected street network to extend their TR. Another explanation might be that well-connected street networks increase proximity to destinations and provide multiple route options as mentioned by Chin, Van Niel, Giles-Corti, and Knuiman (2008), which can encourage children to walk extra-mileage independently. So, it clearly indicates if a neighborhood is characterized by wellconnected and low traffic streets, children tend to explore new discretionary activities in their neighborhoods and therefore increase their TR. As a component of aesthetics and comfort category of BE, a higher proportion of tree shade along routes, which enhances comfort and visual appeal to children as shown in Boldemann et al. (2006), was found to expand overall TR.

Third, the walking environment condition, in general, has been found to have a positive impact on both discretionary and nondiscretionary TRs. However, the impact is two and half times larger on nondiscretionary TR than discretionary TR. In general, these findings imply that if children have the ease of access on the routes that have footpaths, and are free from cracks, holes, dirty objects and obstructions, including poles, garbage vans, vendors, illegally parked vehicles and construction materials, they tend to stretch their TR more. Intuitively, the findings agree with Saelens and Handy (2008)'s finding that a better walking environment correlates with more walking. Poor maintenance or poor physical appearance of a local environment is negatively perceived by children and parents, which in turn, restricts independent travel (Bwire, 2011; Mitra et al., 2014). Thus, a higher proportion of tree shade along routes, which enhances comfort, and visual appeal to children as shown in Boldemann et al. (2006), was found to expand overall TR. The findings imply that policymakers need to think beyond the conventional land uses and street design factors. They also need to think about aesthetic quality, maintenance, and the 
comfort level of the urban environment as important criteria of BE design to promote TR.

\subsection{The influence of perceived environment and socio-demographic factors on TR}

Children's perception of local environment is found to be a determinant of all types of TRs. Consistent with general assumptions, negative perceptions of the local environment and concerns of safety are found to shorten TR (Loebach \& Gilliland, 2014). Thus, if children perceive that the neighborhood is not a safe place and prone to criminal activities, they tend to shrink their TR. In contrast, better perception of the physical condition, aesthetic and maintenance of the walking environment increases children's satisfaction and therefore, positively impacts both nondiscretionary and discretionary TRs. It is found that the amount of tree that is related to the aesthetic of the route and provides shades, as well as ease of movement to children, has a bigger role on TR. However, it impacts discretionary and nondiscretionary TRs differently. If children perceive that there is a larger amount of tree shades in their neighborhood, they travel extra miles to discretionary destinations, whereas, if children perceive that there is a larger amount of tree shades on their routes to school, then they tend to extend their nondiscretionary TRs. Since the discretionary destinations are generally multiple, multidirectional and located all around the neighborhood, the overall tree coverage of neighborhood is important for this type of TR. One the other hand, due to the mandatory nature and a limited number of nondiscretionary destinations, the perception of the amount of tree on the way to school and coaching can encourage them to travel extra miles independently.

The finding on gender is in line with other TR studies; boys have a greater range to both discretionary and nondiscretionary destinations than girls (Villanueva, Giles-Corti, Bulsara, McCormack, et al., 2012). The range difference between boys and girls is larger for nondiscretionary trips. This may have resulted from the general range difference between discretionary and nondiscretionary trips. Parent's age and education level also influence TR. TR extends with the increase of father's age, suggesting that older fathers become more flexible and more confident about a child's ability to roam around independently. Although fathers seem less restrictive than mothers for children's independent mobility in general (Schoeppe et al., 2016), more studies are needed to understand the change of their perception and parenting style with progressing age. Interestingly, higher education of fathers encouraged TR extension. The higher levels of education probably increase awareness among fathers about the need for TR extension as well as CIM. This finding aligns with Schoeppe et al. (2016) in which parents with lower education are more likely to restrict children's independent mobility.

\section{$5 \quad$ Strengths, limitations and conclusions}

One of the unique aspects of this study was the examination of TR between discretionary and non-discretionary destinations. This research finds that the way TR is measured has an impact. When measured separately, nondiscretionary TR was found to be greater than the discretionary and combined TR. A major contribution of this study is a comparison between two types of TR in relation to a wide variety of $\mathrm{BE}$ and socio-demographic factors. It has been found that due to the differences in the nature of discretionary and nondiscretionary trips, the responses to explanatory variables vary considerably. Syntactic measures, considered for the first time in a TR study, show their relevance along with other widely examined BE variables for TR. Another contribution of this study was the use of a walkability tool to collect route-along BE; this method was time-consuming and laborious but provided useful micro-level route data, which were not possible to gather from buffer-approach (e.g., walking environment obstruction) using secondary dataset (e.g., GIS). A further strength is the exploration of children's perceptions 
of their local environment, which has previously been found influential for other CIM measures (children's independent time outside and mobility licence) and compare them between discretionary and nondiscretionary TRs.

Although this study makes a significant contribution to gaining a robust understanding of TR in relation to various factors, several gaps are identified that future studies should take into account. First, the route-level $\mathrm{BE}$ of each attribute was measured by averaging the segment-based BE values of all segments forming a route. However, for more accurate measurement, route-level BE can be measured by the proportion of segment length. Second, this study only examined children's perception of the local BE. Studies have shown that parental perceptions of the BE shape their parenting practices as well as their granting of mobility license (Prezza et al., 2001). As a result, this study calls for future studies to included parental perception of the BE and improve upon the findings presented here. Third, this research developed a list for a walkability audit, due to an absence of developing country-specific walkability audit tools. This tool should be validated and tested for reliability before applying to similar contexts in future. Fourth, this study shows the importance of purpose-specific independent mobility studies. Therefore, other frequently applied CIM measures, such as mobility license and independent time spent outside, should be examined across discretionary and nondiscretionary destinations, applying a similar kind of research design.

Despite the limitations, the findings presented in this research provide insights into the development of TR; a contributor to child's overall physical and mental health along with environmental sustainability and community vibrancy. The children in this study demonstrated longer nondiscretionary TR and shorter discretionary TR at the local level. TR has been found to be influenced by many factors including the purpose of trips, geographic and syntactic BE factors (angular step-depth, presence of residential land use, and commercial and retail land use along the route, walking environment condition, street connectivity and the number of trees), child's gender, parent's age and level of education, and children's perception of the neighborhood (level of crime and amount of trees present in the neighborhood). The overall findings demonstrate that BE factors have the largest impact on TR followed by perceptual factors and sociodemographic factors respectively. These findings are likely to inform the design of childfriendly cities and urban places.

\section{Acknowledgements}

The authors thank the two anonymous reviewers for their insightful comments and suggestions. 


\section{References}

Alparone, F. R., \& Pacilli, M. G. (2012). On children's independent mobility: The interplay of demographic, environmental, and psychosocial factors. Children's Geographies, 10(1), 109-122.

Argin, G., \& Torun, A. O. (2015). Walking to school: The effects of street network configuration and urban design qualities on route selection behavior of elementary school students. Paper presented at the 10th International Space Syntax Symposium, July 2015, London.

Badland, H. M., Opit, S., Witten, K., Kearns, R. A., \& Mavoa, S. (2010). Can virtual streetscape audits reliably replace physical streetscape audits? Journal of Urban Health, 87(6), 1007-1016.

BCA (Building Construction Act). (2008). Building Construction Act RAJUK 2008. Retrieved from https://www.slideshare.net/PriodeepChowdhury/dhaka-imaratnirmanbidhimala2008

Boldemann, C., Blennow, M., Dal, H., Mårtensson, F., Raustorp, A., Yuen, K., \& Wester, U. (2006). Impact of preschool environment upon children's physical activity and sun exposure. Preventive Medicine, 42(4), 301-308.

Bwire, H. (2011). Children's independent mobility and perceptions of outdoor environments in Dar Es Salaam City, Tanzania. Global Studies of Childhood, 1(3), 185-206.

Can, I., \& Heath, T. (2016). In-between spaces and social interaction: A morphological analysis of Izmir using space syntax. Journal of Housing and the Built Environment, 31(1), 31-49.

Carver, A., Panter, J. R., Jones, A. P., \& van Sluijs, E. M. (2014). Independent mobility on the journey to school: A joint cross-sectional and prospective exploration of social and physical environmental influences. Journal of Transport \& Health, 1(1), 25-32. http://doi:10.1016/j.jth.2013.12.003

Chin, G. K. W., Van Niel, K. P., Giles-Corti, B., \& Knuiman, M. (2008). Accessibility and connectivity in physical activity studies: The impact of missing pedestrian data. Preventive Medicine, 46(1), $41-45$.

Collins, D. C. A., \& Kearns, R. A. (2001). The safe journeys of an enterprising school: Negotiating landscapes of opportunity and risk. Health and Place, 7(4), 293-306. http://doi:10.1016/S13538292(01)00021-1

Connolly, K., \& Bruner, J. (1974). The growth of competence (Vol. 1). New York: Academic Press.

De Meester, F., Van Dyck, D., De Bourdeaudhuij, I., \& Cardon, G. (2014). Parental perceived neighborhood attributes: Associations with active transport and physical activity among 10- to 12-yearold children and the mediating role of independent mobility. BMC Public Health, 14(1), 631. http:// doi:10.1186/1471-2458-14-631

Fagan-Watson, B., Shaw, B., Bicket, M., Mocca, E., Elliott, B., \& Hillman, M. (2015). Children’s independent mobility: An international comparison and recommendations for action. London: Policy Studies Institute.

Fagerholm, N. C. \& Broberg, A. (2011). Mapping and characterizing children's daily mobility in urban residential areas in Turku, Finland. Fennia-International Journal of Geography, 189(2), 31-46.

Fyhri, A., Hjorthol, R., Mackett, R. L., Fotel, T. N., \& Kyttä, M. (2011). Children's active travel and independent mobility in four countries: Development, social contributing trends and measures. Transport Policy, 18(5), 703-710.

Gershuny, J. (1993). Escorting children: Impact on parental lifestyle. In Children, transport and the quality of life (pp. 62-76). London: Policy Studies Institute.

Ghekiere, A., Deforcheab, B., Carver, A., Mertens, L., de Geus, B. Clarys, P., . . Van Cauwenberg, J. (2016). Insights into children's independent mobility for transportation cycling - which socioecological factors matter? Journal of Science and Medicine in Sport, 20(3), 267-272.

Helbich, M, van Emmichoven, M. J. Z., Dijst, M. J., Kwan, M.-P., Pierik, F. H., \& de Vries, S. I. (2016). Natural and built environmental exposures on children's active school travel: A Dutch global 
positioning system-based cross-sectional study. Health \& Place, 39, 101-109.

Hillier, B. (1998). A note on the intuiting of form: Three issues in the theory of design. Environment and Planning B: Planning and Design Anniversary Issue, 25(7), 37-40.

Hillier, B. (2007). Space is the machine: A configurational theory of architecture. London: Space Syntax, UCL.

Hillier, B., \& Hanson, J. (1984). The social logic of space, 1984. Cambridge, UK: Press syndicate of the University of Cambridge.

Hillier, B., \& Iida, S. (2005). Network and psychological effects in urban movement. Paper presented at the International Conference on Spatial Information Theory, Berlin.

Hillman, M., Adams, J., \& Whitelegg, J. (1990). One false move. London: Policy Studies Institute.

Jansen-Osmann, P., \& Wiedenbauer, G. (2004). The influence of turns on distance cognition: New experimental approaches to clarify the route-angularity effect. Environment and Behavior, 36(6), 790-813.

Lee, C., Kim, H. J., Dowdy, D. M., Hoelscher, D. M., \& Ory, M. G. (2013). TCOPPE school environmental audit tool: Assessing safety and walkability of school environments. Journal of Physical Activity and Health, 10(7), 949-960.

Loebach, J. E., \& Gilliland, J. A. (2014). Free range Kids? Using GPS-derived activity spaces to examine childrens neighborhood activity and mobility. Environment and Behavior, 48(3), 421-453. http:// doi: $10.1177 / 0013916514543177$

Lopes, F., Cordovil, R., \& Neto, C. (2014). Children's independent mobility in Portugal: Effects of urbanization degree and motorized modes of travel. Journal of Transport Geography, 41, 210-219. http://doi:10.1016/j.jtrangeo.2014.10.002

McDonald, N. C., \& Aalborg, A. E. (2009). Why parents drive children to school: Implications for safe routes to school programs. Journal of the American Planning Association, 75(3), 331-342.

Mitra, R., Faulkner, G. E. J., Buliung, R. N., \& Stone, M. R. (2014). Do parental perceptions of the neighborhood environment influence children's independent mobility? Evidence from Toronto, Canada. Urban Studies, 0042098013519140.

Monsur, M., \& Islam, Z. (2011). Impact of street design on children's independent mobility in Dhaka City. Paper presented at the Emergent Placemaking, Seattle, WA.

Monsur, M., Mansur, M., \& Islam, M. Z. (2017). Are children living on dead-end streets more active? Near-home street patterns and school-going children's time spent outdoors in Dhaka, Bangladesh. Preventive Medicine, 103, S73-S80.

Moore, R., \& Young, D. (1978). Childhood outdoors: Toward a social ecology of the landscape. In Children and the Environment (pp 83-130). New York: Plenum Publishing.

Ozbil, A., Argin, G., \& Yesiltepe, D. (2016). Pedestrian route choice by elementary school students: The role of street network configuration and pedestrian quality attributes in walking to school. International Journal of Design Creativity and Innovation, 4(2), 67-84.

Özbil Torun, A. (2015). Walking to school: The effects of street network configuration and urban design qualities on route selection behavior of elementary school students. Paper presented at the 10th International Space Syntax Symposium, July 2105, London.

Page, A. S, Cooper, A. R., Griew, P., \& Jago, R. (2010). Independent mobility, perceptions of the built environment and children's participation in play, active travel and structured exercise and sport: The PEACH Project. International Journal of Behavioral Nutrition and Physical Activity, 7(1), 1.

Prezza, M., Alparone, F. R., Cristallo, C., \& Luigi, S. (2005). Parental perception of social risk and of positive potentiality of outdoor autonomy for children: The development of two instruments. Journal of Environmental Psychology, 25(4), 437-453. 
Prezza, M., Alparone, F. R., Renzi, D., \& Pietrobono, A. (2009). Social participation and independent mobility in children: The effects of two implementations of "we go to school alone." Journal of Prevention \& Intervention in the Community, 38(1), 8-25.

Prezza, M., Pilloni, S., Morabito, C., Sersante, C., Alparone, F. R., \& Giuliani, M. V. (2001). The influence of psychosocial and environmental factors on children's independent mobility and relationship to peer frequentation. Journal of Community \& Applied Social Psychology, 11(6), 435-450. http:// doi:10.1002/casp.643

Ratti, C. (2004). Space syntax: Some inconsistencies. Environment and Planning B: Planning and Design, 31(4), 487-499.

Saelens, B. E., \& Handy, S. L. (2008). Built environment correlates of walking: A review. Medicine and Science in Sports and Exercise, 40(7), S550.

Sallis, J. F., Cain, K. L., Conway, T. L., Gavand, K. A., Millstein, R. A., Geremia, C. M., . . King, A. C. (2015). Peer reviewed: Is your neighborhood designed to support physical activity? A brief streetscape audit tool. Preventing Chronic Disease, 12, e141.

Schoeppe, S., Duncan, M. J., Badland, H. M., Rebar, A. L., \& Vandelanotte, C. (2016). Too far from home? Adult attitudes on children's independent mobility range. Children's Geographies, 14(4), 482-489.

Sharmin, S., \& Kamruzzaman, M. (2017). Association between the built environment and children's independent mobility: A meta-analytic review. Journal of Transport Geography, 61, 104-117.

Sharmin, S., \& Kamruzzaman, M. (2018a). The impacts of syntactic measures on pedestrian movement: $A$ comparative meta-analytic review between developing and developed countries. Paper presented at the State of Australian Cities Conference, November 2017, Adelaide, Australia.

Sharmin, S., \& Kamruzzaman, M. (2018b). Meta-analysis of the relationships between space syntax measures and pedestrian movement. Transport Reviews, 38(4), 524-550.

Sharmin, S., Kamruzzaman, M., \& Haque, M. M. (2020). The impact of topological properties of built environment on children independent mobility: A comparative study between discretionary vs. nondiscretionary trips in Dhaka. Journal of Transport Geography, 83, 102660.

Shaw, B., Watson, B., Frauendienst, B., Redecker, A., Jones, T., \& Hillman, M. (2013). Children's independent mobility: A comparative study in England and Germany (1971-2010). London: Policy Studies Institute.

Spilsbury, J. C. (2005). 'We don't really get to go out in the front yard'-children's home range and neighborhood violence. Children's Geographies, 3(1), 79-99.

Timperio, A., Crawford, D., Telford, A., \& Salmon, J. (2004). Perceptions about the local neighborhood and walking and cycling among children. Preventive Medicine, 38(1), 39-47.

Tranter, P., \& Doyle, J. (1996). Reclaiming the residential street as play space. International Play Journal, 4, 81-97.

Tranter, P., \& Pawson, E. (2001). Children's access to local environments: A case-study of Christchurch, New Zealand. Local Environment, 6(1), 27-48.

Tranter, P., \& Whitelegg, J. (1994). Children's travel behaviors in Canberra: Car-dependent lifestyles in a low-density city. Journal of Transport Geography, 2(4), 265-273. http://doi:10.1016/09666923(94)90050-7

U.S. Environmental Protection, Agency. (2003). Travel and environmental implications of school siting [electronic resource]. Retrieved from https://www.epa.gov/smartgrowth/travel-and-environmentalimplications-school-siting

Van Vliet, W. (1983). Exploring the fourth environment: An examination of the home range of city and suburban teenagers. Environment and Behavior, 15(5), 567-588. 
Veitch, J., Salmon, J., \& Ball, K. (2008). Children's active free play in local neighborhoods: A behavioral mapping study. Health Education Research, 23(5), 870-879.

Villanueva, K., Giles-Corti, B., Bulsara, M., McCormack, G. R., Timperio, A., Middleton, N., . . . Trapp, G. (2012). How far do children travel from their homes? Exploring children's activity spaces in their neighborhood. Health Place, 18(2), 263-273.

Villanueva, K., Giles-Corti, B., Bulsara, M., Timperio, A., McCormack, G., Beesley, B., . . Middleton, N. (2012). Where do children travel to and what local opportunities are available? The relationship between neighborhood destinations and children's independent mobility. Environment and Behavior, 45(6), 679-705. http://doi:10.1177/0013916512440705

Villanueva, K., Giles-Corti, B., Bulsara, M., Trapp, G., Timperio, A., McCormack, G., \& Van Niel, K. (2013). Does the walkability of neighborhoods affect children's independent mobility, independent of parental, socio-cultural and individual factors? Children's Geographies, 12(4), 393-411. http://doi $: 10.1080 / 14733285.2013 .812311$

Xia, X. (2013). A comparison study on a set of space syntax based methods: Applying metric, topological and angular analysis to natural streets, axial lines and axial segments. (Masters thesis), University of Gävle, Gävle, Sweden.

Zdrahalova, J., \& Boumova, I. (2016). Social and environmental factors influencing active school transport (AST) in Radotin, Prague. Paper presented at the Smart Cities Symposium Prague (SCSP) 2016. 